\section{Reliable and innovative blocs}

As CAD/CAM technology continues to be integrated into dental practices, the benefits of its use cannot be overstated. Dental $\mathrm{CAD} / \mathrm{CAM}$ systems and relevant materials allow clinicians to offer a variety of innovative dental services to many of their patients who otherwise may receive less than optimal care, or require multiple and time-consuming appointments.

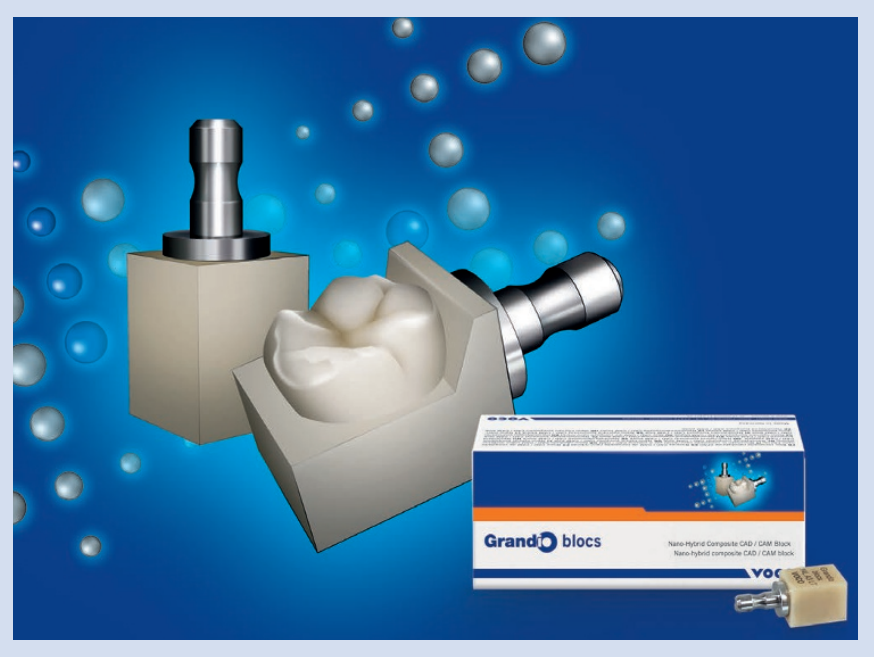

CAD/CAM technology can be used to design and fabricate both provisional and definitive restorations with great ease and the process becomes even more predictable when advanced materials such as VOCO nano-hybrid Grandio blocs are used.

As the strongest in its class, with a superior biaxial flexural material strength of $333 \mathrm{MPa}$ and a filler content of $86 \%$ by weight, these pioneering Grandio blocs boast the highest filler content of any bloc on the market today. Highly-regarded for their aesthetic surface finish, Grandio blocs are a genuine alternative to ceramics for same-day chairside CAD/CAM restorations. They offer a whole range of additional benefits, from outstanding physical values, fracture and abrasion resistance coupled with exceptional colour characteristics to perfectly restore aesthetics, function and morphology.

Combining proven nano-hybrid Grandio technology with CAD/ CAM processing results in a block that is easy to work with, offers strength and biocompatibility and guarantees outstanding, lasting aesthetic results.

To find out why VOCO is a trusted partner in restorative dentistry call the expert VOCO team today free on 0080044444 555 or email service@voco.de.

www.voco.com

\section{Digital symposium a huge success}

A gathering of more than 400 delegates at this year's Henry Schein ConnectDental Digital Symposium in Central London is testament to just how far the digital message is resonating with the profession.

The diverse two-day programme covered all aspects of the digital spectrum, through a series of lectures, presentations and break-out sessions and was opened with a keynote lecture by Ian Buckle. $\mathrm{Mr}$ Buckle examined the important relationship between clinicians and their technical colleagues and how the use of digital tools can aid communication not only with the laboratory, but also other specialists, including orthodontists, implant dentists and surgeons.

The Henry Schein ConnectDental platform offers digital technology solutions to help improve efficiency and productivity for both the practice and the laboratory with the aim of enhancing the patient experience by delivering predictable, high quality dentistry. The role of digital dentistry in achieving this aim was perfectly illustrated by a two-part presentation by Colin Campbell and Jameel Gardee, which included a full case study on digitally planning the smile and guided implant surgery. The presentation included details of how intraoral scanning, CBCT, digital X-rays, digital photography and smartphone video were all used to provide a fully-planned, interdisciplinary case, which included the live capture of a patient's information. In addition, the case demonstrated how to create a digital wax-up as well as the production of a milled implant surgical guide. The case was completed on the Saturday morning when Colin described the surgical procedures and compared the relative benefits of the different guided systems used on the case.

With this symposium, Henry Schein guided those taking their first steps in the digital world and assisted those practitioners who have already embraced the technology and are seeking to perfect their craft.

\section{New cosmetic dental information site}

My White Clinic launched their new cosmetic dentistry information website at the Scottish Dental show in May with a lot of interest. The website MyWhiteClinic.com is for patients to use as an educational resource which helps to generate interest and the take-up of private cosmetic dental treatments. As the website features a range of educational videos with fast easy navigation it can be a useful tool for dental professionals who are members.

The educational information on the site helps new and existing patients to feel more confident to discuss new treatments and has generated, in a six month pilot test at an independent mixed practice, an

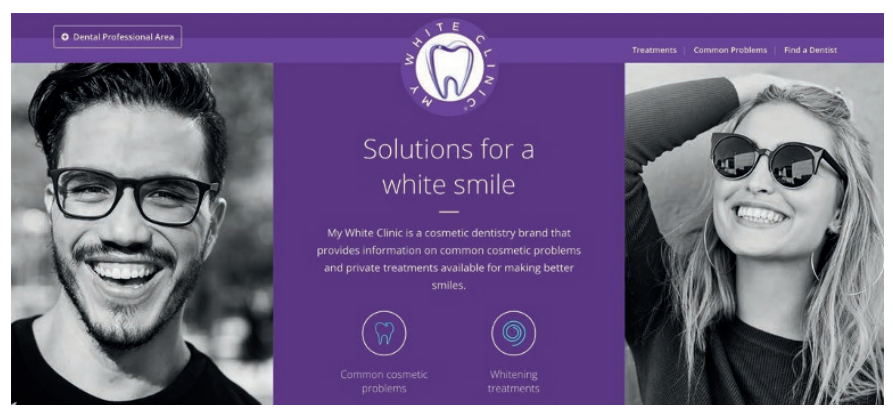

increased take-up of private cosmetic dental treatments.

Dental professionals can join on the website in minutes and pay a small monthly fee. A key benefit of joining is an exclusive listing on mywhiteclinic.com which means members will be the only one search result in their postcode. Take a tour of mywhiteclinic.com - in the dental professional area you can find out more about member benefits.

The website was developed by My White Clinic ltd as a showcase for their signature easy navigation and CQC compliant website development service - for more details email info@mywhiteclinic.com. 